\title{
RESEARCH ON THE DESIGN AND CONTROL OF ADVANCED EXPERIMENTAL SETUPS USED IN ACTUATORS AND PLATFORMS CHARACTERIZATION
}

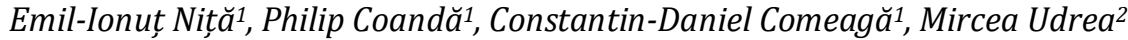 \\ 1 University "Politehnica" of Bucharest \\ ${ }^{2}$ Apel Laser S.R.L \\ Email: philip.coanda@upb.ro
}

\begin{abstract}
The current paper proposes an enhanced 4 DOF measurement platform designed to automatically determine the static and dynamic characteristics of electromagnetic and piezo actuators. High precision motor or laser instruments can be attached on the given platform, providing a high level of versatility for multiple applications. Further, the constructive and design parameters of the platform will be discussed in detail with some applications proposed. A control algorithm and a demo LabVIEW program is proposed as well based on the given application, analysing an electromagnet actuator.
\end{abstract}

Keywords: Actuators Characterization, myRIO control, Linear Motor Control, Positioning, LabVIEW.

\section{Introduction}

As every design requires some measurements to be performed, during the process and at the end, for product validation, high reliability measurement platforms are a key element in the designing process [1], [2], [3]. Moreover, if the platform is designed to be modular [4], it can be easily adapted to different type of measurements, giving it a higher level of usability. Actuator's measurements have high importance in domains where customer safety is first in the line such as medicine (surgery laser devices [5]), automotive (braking system [6]) or aviation [7].

In order to proceed with the design of the platform, a clear overview of the actuators, systems or components which are considered for measurement is needed, as parameters like size, load and control are highly dependent on application. The platform's design was developed considering the need of measurements in the following fields:

- Characterization of piezoelectric actuators

- Characterization of electrodynamic actuators

- Characterization of electromagnetic actuators

- Characterization of custom designed positioning subassemblies

The proper design and functioning of this type of actuators depend on reliable measurements taken with high precision equipment and powerful software.
A review of the literature underlines the need of actuators characterization, to optimize the designing, manufacturing, and validation process [8], [9], [10].

\section{Characterizations to be Considered}

As mentioned since the introduction, the experimental setup aims to be capable to perform measurements and characterization for a wide range of actuators and micro actuators, but also for subassemblies used in positioning applications.

The measurements in question can be performed both in static and dynamic setups, depending on the needs, as well as depending on the availability of the proper measurement tools and control elements. Given the necessities, the types of possible actuator characterizations are:

- Static measurements:

- Characterization of the rigidity of the actuators or assemblies.

- Static response at a given input signal

- Dynamic measurements:

- Mechanical impedance characterization (mass, rigidity and dumping).

- Electrical impedance characterization (resistance, inductance, and capacitance).

Those type of characterization are widely used in design and manufacture of custom actuators, so a proposal for an "all in one", adaptive measurement experimental setup is relevant to industry 


\section{Static Characterization of Actuators}

This type of characterization is relatively simple to perform, given that it requires a voltage or current control, depending on the type of actuator used and a measurement of displacement or force. Depending on the approach chosen for the control (voltage or current) as well as the measured quantity, the determinations for displacement and force, respectively, can be made in several ways.

Displacement measurements can be done either with an inductive or capacitive position transducer, this being the classic and simplest method to approach, or with a zero transducer. In the second case, when using a zero transducer, a series of motors capable of working on the axes of interest is needed to bring the object to the "zero" position. This method is much more accurate but the rigors of implementation increase.

The determination of the force can also be done in two ways, either in semi-blocking mode or in blocking mode - the ideal case - where the force transducer rigidity is considered to be infinite. In this second case, the transducer is pushed into the part or assembly to be measured with the help of motors whose position can be controlled and measured, and thus the maximum force that is applied to the rigid is determined.

\section{Dynamic Characterization of Actuators}

Dynamic characterization usually implies the use of a professional and properly calibrated signal source and an amplifier to power the actuators. For this type of characterization, a swept signal is applied to the actuators in question, measuring its response as long as it is powered. The output parameter (displacement or acceleration) can be determined either with a laser vibrometer or with a classic contact accelerometer. Optionally, at the base of the stand can be placed a vibration exciter on which is placed the element whose determination is desired to be made. In this case, two measuring points are needed, one for the exciter's table considered the reference position, and one located on the actuator's output.

\section{Structural Design of the Platform}

The platform is designed around two main subassemblies. For easier identification of the two subassemblies, they were named as follows:

- The fixed subassembly, needed to support the second subassembly and to also provide one vertical axis of movement.

- The mobile subassembly which can be configured to have multiple DOF.

Figure 1 shows the two 3D designed subassemblies. The fixed subassembly represents besides the main support element also one of the means by which the guidance for the mobile subassembly is made. The latter can also be modified and adapted according to needs, being possible to add various elements necessary in making measurements to characterize the actuators. Moreover, the mobile subassembly can be composed of several subassemblies, to further expand the capabilities of the measuring stand.

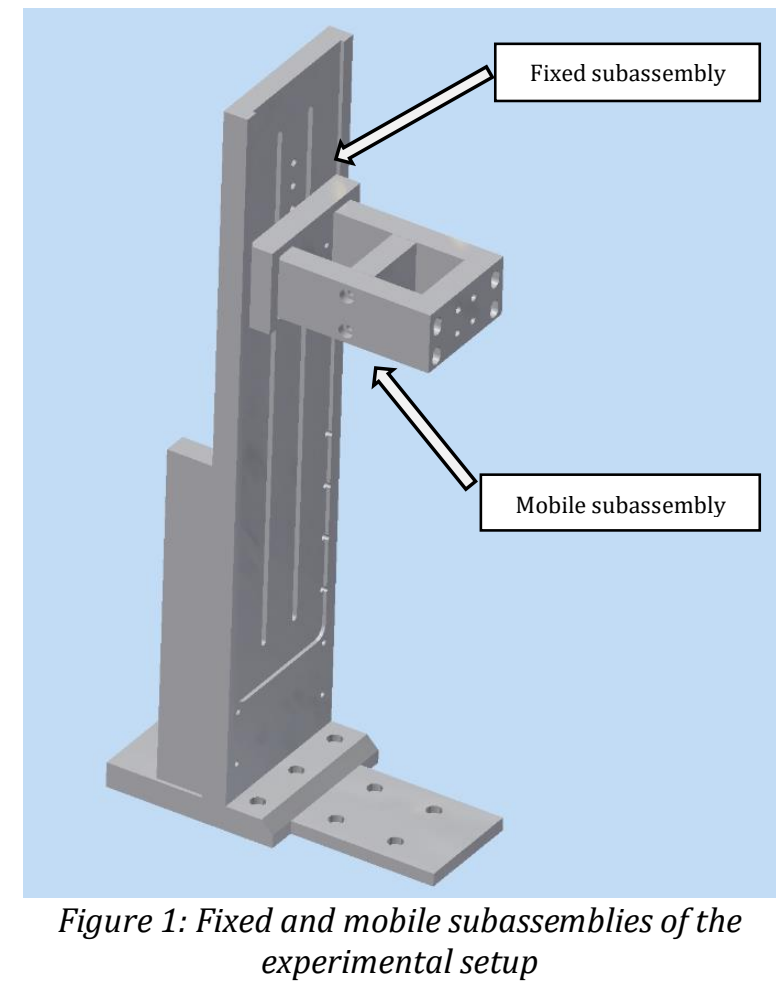

Further, the two subassemblies will be analyzed each, with constructive explanations, and an example of practical application developed in the laboratory will also be provided.

\section{Fixed subassembly}

As can be seen in Figure 1, the fixed subassembly is the basic element of the proposed experimental setup, which is designed to be able to accommodate the mobile subassembly, and also to be able to adapt in various situations of use, having different points where screws or bolts can be inserted.

It was designed from 3 distinct pieces, as follows:

- A metallic support that is used to stiffen the assembly and to allow it to be attached to various surfaces.

- A vertical guide where the mobile subassembly is mounted and which, in addition to the supporting role of the mobile subassembly, can also be used as a vertical translation axis.

- Two vertical guide supports to provide extra stiffness and good support of the guide and the mobile subassembly.

\section{Mobile subassembly}

The mobile subassembly can be seen in Figure 1 also. Considering its constructive characteristics, it was designed to be able to adapt and accommodate to different measurement devices and methods 
relevant for the application in question such as high precision X-Y-Z platforms that can be electrically actuated or manually actuated. To preserve the parallelism from the lower platform or from the actuator under test, an orientation platform (pitch, yaw, roll) can be added.

\section{Mechanical Adaptation of the Proposed Experimental Setup for the Characterization of Electromagnetic Actuators}

As mentioned from the previous chapters, the main feature of the experimental setup in question is the flexibility and ease with which it can be adapted to perform measurements and characterization of specific actuators.

The current example deals with the adaptation of the experimental setup for the characterization of the electromagnetic actuators, and also with the additional elements needed for this characterization. Moreover, the control mode and measurement steps needed for the characterization will be studied.

The main goal is to move the electromagnetic actuator over a permanent magnet, with known structural parameters, to raise the current, force and displacement characteristic.

To achieve the proposed goal, from a structural and functional point of view, the experimental stand was adapted according to Figure 2.

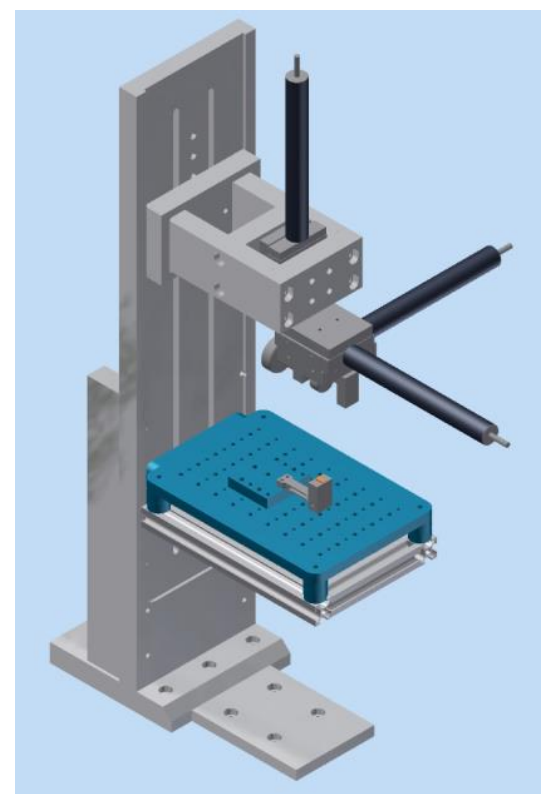

Figure 2: Adaptation of the experimental setup for the characterization of electromagnetic actuators - top view

The top view of the platform highlights the additional movable table connected to the fixed platform, as a support element for the force transducer was needed. To be able to measure the electromagnetic interaction force between the electromagnet and the permanent magnet, on the movable platform was placed a $100 \mathrm{~g}$ load cell with resistive stain gauges, that holds the permanent magnet and its support. Figure 3 presents a closeup of the additional designed table.

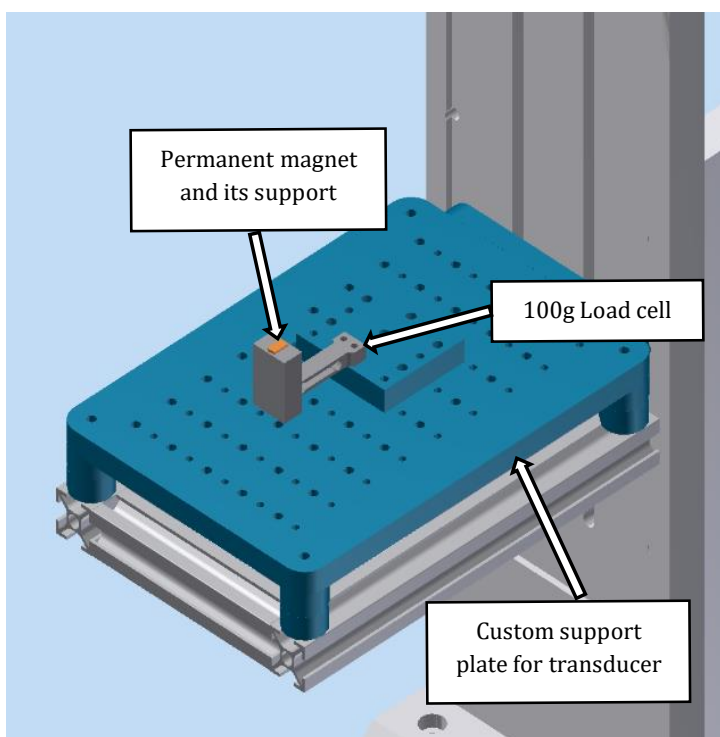

Figure 3: Custom designed table and support for the permanent magnet and load cell

Regarding the mobile subassembly, a series of clamping, guiding and control elements have been added, necessary to be able to hold and move the electromagnet over the permanent magnet.

Because the actuator can be used in 3DOF medical laser platforms [11] where the movable part contains the permanent magnets, this approach was preferred. Therefore, to ensure a very high precision measurement with respect to real life application, the proposed measurement platform must measure the force applied on the permanent magnet side as a function of the current through the electromagnet and the distance between it and the magnet.

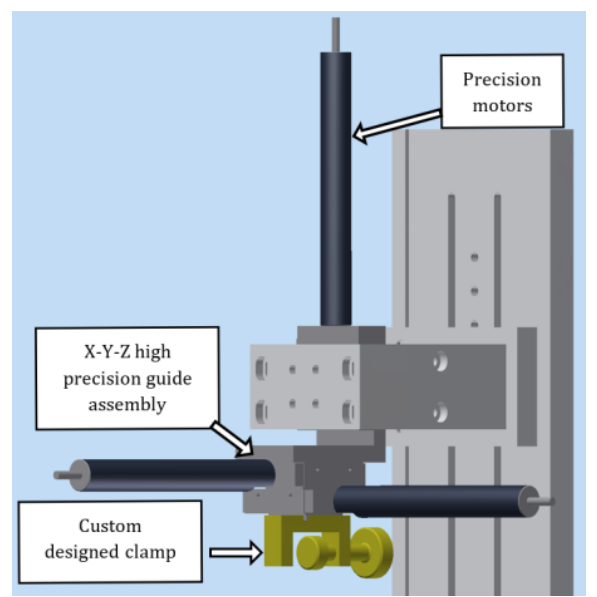

Figure 4: Adaptation of the mobile subassembly by adding multiple elements of clamping, guiding and control 
Figure 3 presents the main items added to the mobile subassembly are:

- 3 M-105 microtranslation stages connected in a XYZ configuration

- $\quad$ High precision DC linear motors M-231.17 connected to the platforms

- 3D printed custom designed PLA clamp

The 3 axes with micrometric precision can be controlled both manually or using M-231.17 High resolution linear motors when the application requests a digitally controlled positioning or closed loop control elements are involved within the design. The 3D printed clamp has the role to grip the electromagnetic actuator and its wire, and it is used as an electromagnetic barrier between the electromagnetic field produced by the actuator (or the magnet) and the other ferromagnetic components.

\section{Control Algorithm and LabVIEW Program for the Electromagnet Characterization}

To control the displacement of the micro translation stages, respectively the displacement of the electromagnet over the permanent magnet, to receive data from the force transducer and to control the electrical current through the actuator, a myRIO 1900 controller was added to the platform. The connection and control diagram of the proposed platform is presented in Figure 5.

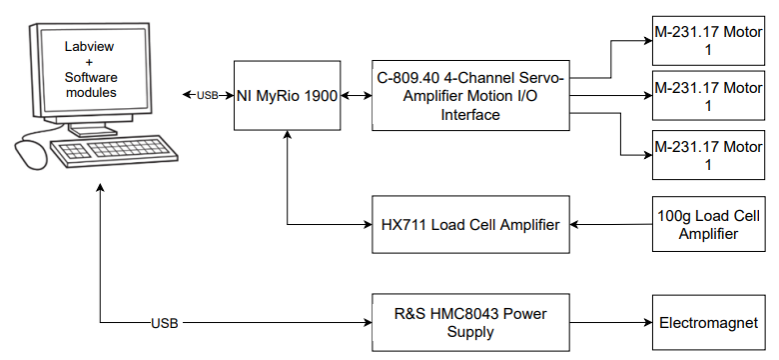

Figure 5: Hardware connection schematics

As can be seen in Figure 5, the NI myRIO 1900 development board has been connected to a PC (main processor) via a USB port. This board will take care of the local control of the M-231.17 DC motors, via the I/O control interface C-809.40 wich delivers the required power for the motors. For each motor, the myRIO 1900 board monitors the stroke limit switches (Low and High), reads the signal from the quadrature encoder (the feedback signal used for the close loop) and generates a voltage control signal in the range $(-10,10 \mathrm{~V})$ depending on a reference position entered by the user from the PC keyboard.
Moreover, NI myRIO 1900 development board takes care of the date acquisition from the resistive force transducer, connected to a 24-Bit Analog-to-Digital Converter (ADC) for Weigh Scales, HX711. To be able to communicate with the digital interface of the HX711, the FPGA module integrated in myRIO was used, in order to be able to respect the timing of the data signal according to the protocol described in the technical data sheet.

The second USB connection establish the communication between the main core LabVIEW program and the R\&S HMC8043 3-channel power supply that can deliver up to $100 \mathrm{~W}$.

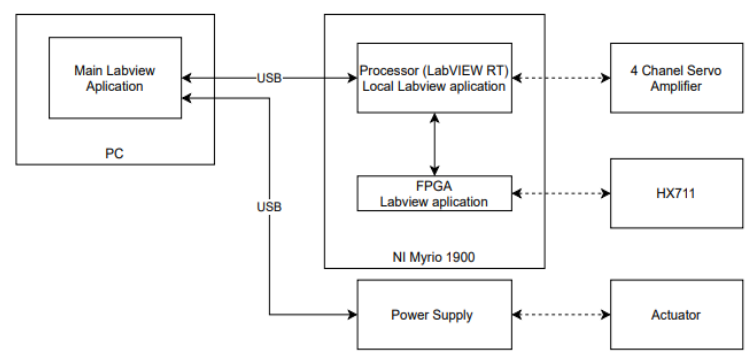

Figure 6: VIs communication between processors diagram

The proposed application uses multiple protocols and processors (Figure 6); therefore, the hole LabVIEW application was divided in multiples VI programs:

- Main LabVIEW program that runs on the PC processor and takes care of the power supply control and manipulates input data from the user interface

- Local LabVIEW program that runs on the myRIO processor, Xilinx Zynq-7010, and takes care of the positioning close loop of the DC motors, process the data received from FPGA module, and communicates with the PC over the UART protocol.

- FPGA LabVIEW application that runs on the FPGA module and takes care of the raw data received from HX711 Analog-to-Digital Converter and send it to the myRIO processor

This configuration was preferred to reduce the computational power over the myRIO processor, and to be able to perform real time measurements.

The main LabVIEW program holds the user interface where all the parameters cand be configured (figure 7). Moreover, the program interface let the user to set the speed of the motors, the absolute position, the power signal sent to the actuator (in this case the total current through the 
coil) and shows the processed and raw data from the force transducer. After the test, the main program saves automatically all the data in name.cvs file and disk location indicated by the user at the beginning of the run.

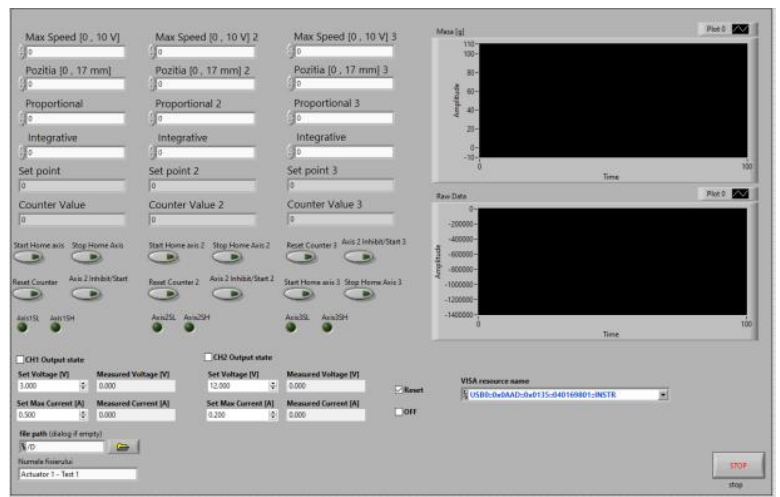

Figure 7: Main program user interface

The implementation of the proposed platform is presented in Figure 8, connected to an electromagnetic actuator for testing.

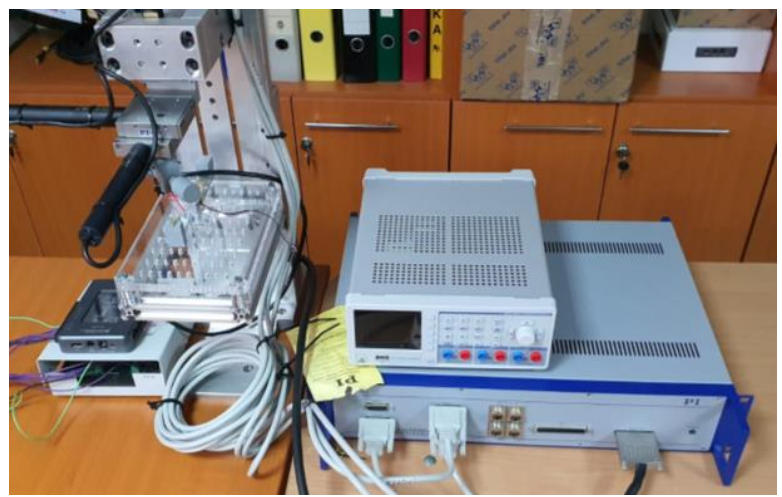

Figure 8: 4DOF measuring platform

\section{Conclusions}

A 4 DOF measurement platform was presented in this paper, designed to automatically determine the current-force-displacement of an electromagnetic actuator. The mechanical structure and its flexibility for developing other actuator testing platforms was explained along with the main application structure and its user interface.

Further research will focus on multiple types of testing using a laser vibrometer and a shaking table.

\section{Acknowledgements}

This work was supported by a grant of the Romanian Ministry of Education and Research, CCCDIUEFISCDI, project number PN-III-P2-2.1-PTE-20190253, within PNCDI III.

\section{References}

[1] A. Lovisolo, P. E. Roccato, și M. Zucca, „Analysis of a magnetostrictive actuator equipped for the electromagnetic and mechanical dynamic characterization", J. Magn. Magn. Mater., vol. 320, nr. 20, pp. e915-e919, oct. 2008, doi: 10.1016/j.jmmm.2008.04.065.

[2] S. G. Sawant, M. Oyarzun, M. Sheplak, L. N. Cattafesta, și D. P. Arnold, „Modeling of Electrodynamic Zero-Net Mass-Flux Actuators", AIAA J., vol. 50, nr. 6, pp. 1347-1359, 2012, doi: 10.2514/1.J051459.

[3] S. G. Sawant, N. Wang, M. S. Hanna, C. R. Taylor, și D. P. Arnold, „Fabrication, Characterization, and Modeling of Fully-Batch-Fabricated Piston-Type Electrodynamic Microactuators", J. Microelectromechanical Syst., vol. 23, nr. 1, pp. 220-229, feb. 2014, doi: 10.1109/JMEMS.2013.2271293.

[4] Pier C. B.,Matteo D. L., Paolo M. , „Design and Development of an Electromechanical Actuator Test Bench for Validation of Health Monitoring Models" Conference: 31st European Safety and Reliability Conference (ESREL 2021), doi: 10.3850/978-981-18-2016-8_560-cd

[5] Takafumi 0. Toshio 0. Katsumi S. „History of Medical Laser Devices and Recommendations for Clinical Use of Laser Devices", Nippon Laser Igakkaishi 36(4):494-498, doi: 10.2530/ jslsm.jslsm -36_0047

[6] S. Hamsini, M. Kathiresh, „Automotive Safety Systems”, EAI/Springer Innovations in Communication and Computing (2021) doi: 10.1007/978-3-030-59897-6_1

[7] Clarence C. R. „Aviation Safety: Commercial Airlines”, International Encyclopedia of Transportation 2021, Pages 90-97, doi: 10.1016/b978-0-08-102671-7.10113-7

[8] C. Zhi et al., „Fabrication and characterization of micro electromagnetic linear actuators", J. Micromechanics Microengineering, vol. 30, nr. 12, p. 125011, nov. 2020, doi: 10.1088/13616439/abc31e.

[9] X. Wang și Y. Shen, „On the characterization of piezoelectric actuators attached to structures", Smart Mater. Struct., vol. 7, nr. 3, pp. 389-395, iun. 1998, doi: 10.1088/0964-1726/7/3/013.

[10] V. Giurgiutiu și A. N. Zagrai, „Characterization of Piezoelectric Wafer Active Sensors", J. Intell. Mater. Syst. Struct., vol. 11, nr. 12, pp. 959-976, dec. 2000, doi: 10.1106/A1HU-23JD-M5AUENGW.

[11] E.I.Nita, P. Coanda, C.D. Comeaga, M. Udrea, „Active tremor compensation for laser medical instruments”, IJOMAM 2021 Issue9. 